\title{
Crystalline and magnetic structures, magnetization, heat capacity, and anisotropic magnetostriction effect in a yttrium-chromium oxide
}

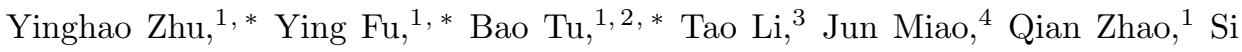 \\ Wu ${ }^{1}$ Junchao Xia, ${ }^{1}$ Pengfei Zhou, ${ }^{1}$ Ashfia Huq,${ }^{5}$ Wolfgang Schmidt, ${ }^{6}$ Defang

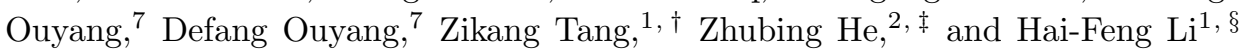 \\ ${ }^{1}$ Joint Key Laboratory of the Ministry of Education, \\ Institute of Applied Physics and Materials Engineering, University of Macau, \\ Avenida da Universidade, Taipa, Macao SAR 999078, China \\ ${ }^{2}$ Department of Materials Science and Engineering, \\ Shenzhen Key Laboratory of Full Spectral Solar Electricity Generation (FSSEG), \\ Southern University of Science and Technology, No. 1088, \\ Xueyuan Rd., Shenzhen 518055, Guangdong, China \\ ${ }^{3}$ Neutron Scattering Technical Engineering Research Center, School of Mechanical Engineering, \\ Dongguan University of Technology, Dongguan 523808, China \\ ${ }^{4}$ School of Materials Science and Engineering, University of Science and Technology Beijing, Beijing 100083, China \\ ${ }^{5}$ Oak Ridge National Laboratory, Oak Ridge, Tennessee 37831, USA \\ ${ }^{6}$ Forschungszentrum Jülich GmbH, Jülich Centre for Neutron Science at ILL, 71 avenue des Martyrs, Grenoble 38042, France \\ ${ }^{7}$ State Key Laboratory of Quality Research in Chinese Medicine, \\ Institute of Chinese Medical Sciences (ICMS), University of Macau, \\ Avenida da Universidade, Taipa, Macao SAR 999078, China
}

(Dated: September 22, 2020)

\begin{abstract}
We have studied a nearly stoichiometric insulating $\mathrm{Y}_{0.97(2)} \mathrm{Cr}_{0.98(2)} \mathrm{O}_{3.00(2)}$ single crystal by performing measurements of magnetization, heat capacity, and neutron diffraction. Albeit that the $\mathrm{YCrO}_{3}$ compound behaves like a soft ferromagnet with a coersive force of $\sim 0.05 \mathrm{~T}$, there exist strong antiferromagnetic (AFM) interactions between $\mathrm{Cr}^{3+}$ spins due to a strongly negative paramagnetic Curie-Weiss temperature, i.e., $-433.2(6) \mathrm{K}$. The coexistence of ferromagnetism and antiferromagnetism may indicate a canted AFM structure. The AFM phase transition occurs at $T_{\mathrm{N}}=141.5(1)$ $\mathrm{K}$, which increases to $T_{\mathrm{N}}(5 \mathrm{~T})=144.5(1) \mathrm{K}$ at $5 \mathrm{~T}$. Within the accuracy of the present neutrondiffraction studies, we determined a G-type AFM structure with a propagation vector $\mathbf{k}=\left(\begin{array}{lll}1 & 1 & 0\end{array}\right)$ and $\mathrm{Cr}^{3+}$ spin directions along the crystallographic $c$ axis of the orthorhombic structure with space group Pnma below $T_{\mathrm{N}}$. At $12 \mathrm{~K}$, the refined moment size is $2.45(6) \mu_{\mathrm{B}}, \sim 82 \%$ of the theoretical saturation value $3 \mu_{\mathrm{B}}$. The $\mathrm{Cr}^{3+}$ spin interactions are probably two-dimensional Ising like within the reciprocal $\left(\begin{array}{lll}1 & 1 & 0\end{array}\right)$ scattering plane. Below $T_{\mathrm{N}}$, the lattice configuration $(a, b, c$, and $V)$ deviates largely downward from the Grüneisen law, displaying an anisotropic magnetostriction effect and a magnetoelastic effect. Especially, the sample contraction upon cooling is enhanced below the AFM transition temperature. There is evidence to suggest that the actual crystalline symmetry of $\mathrm{YCrO}_{3}$ compound is probably lower than the currently assumed one. Additionally, we compared the $t_{2 \mathrm{~g}}$ $\mathrm{YCrO}_{3}$ and the $e_{\mathrm{g}} \mathrm{La}_{7} \mathrm{Sr}_{1 / 8} \mathrm{MnO}_{3}$ single crystals for a further understanding of the reason for the possible symmetry lowering.
\end{abstract}

\section{INTRODUCTION}

Magnetic materials with ferroelectricity are very interesting because a spontaneous electric polarization exists within them to give the substance extraordinary physical and electronic properties and a wide variety of applications such as data storage, catalysts, fuel cells, and sensors 1 - 3. The magnetic and ferroelectric properties can be coupled with each other, therefore, an applied magnetic/electric field is able to switch the electric polarization/magnetization. Above the ferroelectric phase transition, the ferroelectric materials are usually centrosymmetric structurally and behave as an ordinary dielectric; below the phase transition, an electrically-polarized phase forms spontaneously with a noncentrosymmetric structure in the weakly-coupled systems [4] or can be induced by a magnetic phase transition in the strongly- coupled families [5, 6]. Therefore, to build the actual structural and magnetic models may shed light on the nature of the ferroelectric phase transition.

Most ferroelectric materials are perovskite-based oxides. In 1954, Looby and Katz replaced lanthanum in $\mathrm{LaCrO}_{3}$ compound with yttrium during searching for new perovskite-type families and synthesized the $\mathrm{YCrO}_{3}$ compound with an impurity of $\sim 2.5 \% \mathrm{Cr}_{2} \mathrm{O}_{3}$ using $\mathrm{NaCl}$ as the flux under a hydrogen atmosphere [7]. Based on the observation of a very weak extra Bragg peak, they indexed the x-ray powder-diffraction pattern with a monoclinic cell $(a=c=7.61 \AA$, and $b=7.54 \AA)$ by doubling the fundamental perovskite unit cell [7]. One year later, the crystal structure (Fig. 1) was determined to be orthorhombic (with space group Pbnm) with unitcell constants $a=5.238, b=5.518$, and $c=7.54 \AA[8$. This structure becomes more distorted as the pressure in- 
creases [9]. The lanthanide orthochromites of general formula $\mathrm{RECrO}_{3}(\mathrm{RE}=$ rare earth and $\mathrm{Y})$ can be prepared by four different self-propagating high-temperature syntheses [10], i.e., the amorphous citrate precursor method [11, the conventional solid-state reaction method [1214], the hydrothermal synthesis [15], and the microwaveassisted technique [16. Chemical substitution effect in the $\mathrm{Y}_{1-\mathrm{x}} \mathrm{M}_{\mathrm{x}} \mathrm{CrO}_{3}(\mathrm{M}=\mathrm{Mg}, \mathrm{Ca}, \mathrm{Sr}, \mathrm{Ba})$ compound 17. and defect chemistry of the Ca-doped $\mathrm{YCrO}_{3}$ compound [18. were investigated. The $\mathrm{YCrO}_{3}$ compound in forms of bulk and thin film was suggested to be a candidate material for high-temperature thermistors [19 21]. Among the catalysts of $\mathrm{ABO}_{3}$-type perovskite oxides $(\mathrm{A}=\mathrm{La}$, $\mathrm{Y}, \mathrm{Nd}, \mathrm{Gd} ; \mathrm{B}=\mathrm{Fe}, \mathrm{Mn}, \mathrm{Cr}, \mathrm{Co}$ ) for the oxidation of 1,2-dichlorobenzene, the $\mathrm{YCrO}_{3}$ compound was found to be the most active catalyst and was the only one that displayed no loss of its initial activity after several hours on stream [11. The studies of nanocrystalline (un)doped $\mathrm{YCrO}_{3}$ materials were reported 22 24]. The Nd-doped $\mathrm{YCrO}_{3}$ nanoparticles display a semiconducting feature and an enhanced dc conductivity as the Nd content increases, following the Dyre's free energy barrier model 24. The magnetic configuration of the $\mathrm{YCrO}_{3}$ compound below $T_{\mathrm{N}}=140 \mathrm{~K}$ was proposed to be a canted antiferromagnetic (AFM) structure with antisymmetric spin superexchanges 25 27. It was reported that there existed a spin reorientation of the $\mathrm{Cr}^{3+}$ moments in the $\mathrm{YCrO}_{3}$ compound at $\sim 60 \mathrm{~K}$, corresponding to a rotation of the AFM easy axis 28. Ferrimagnetism was found in the half-doped $\mathrm{YMn}_{0.5} \mathrm{Cr}_{0.5} \mathrm{O}_{3}$ compound [29. The magnetic interactions in bulk $\mathrm{YCrO}_{3}$ compound were classified as classical three-dimensional isotropic Heisenberg universality according to the $a b$ initio calculations and Monte Carlo simulations based on a cubic structure with space group Pm3m and lattice constant $a=3.76 \AA$ [30. To understand the ferroelectric anomaly occurring at $\sim 473 \mathrm{~K}$, the first-principles density functional theory calculations found that the noncentrosymmetric monoclinic structure (with space group $P 2_{1}$ ) was the stablest one in view of its lowest energy 31 . The $\mathrm{YCrO}_{3}$ compound was reported to be a relaxor ferroelectric material at about $450 \mathrm{~K}$ because of the local noncentrosymmetric structure 28]. The high-temperature magnetism (300$980 \mathrm{~K}$ ) and crystallographic information (321-1200 K) were studied by a time-of-flight neutron powder diffraction [14, and it is of great interest that the structural information such as lattice constants, space group, bond angles, bond lengths, and the local distortion parameter have no response to the dielectric anomaly observed around $473 \mathrm{~K}$ [31. There has been a long-standing debate about the decision as to which structural symmetry is correct $7-38$, which necessitates a growth of the highquality $\mathrm{YCrO}_{3}$ single crystal 3438 and a careful study of its crystalline and magnetic properties. Previously, small yttrium chromite single crystals with millimeter in size were grown from the $\mathrm{PbF}_{2}-\mathrm{B}_{2} \mathrm{O}_{3}$ or the $\mathrm{PbF}_{2}-\mathrm{B}_{2} \mathrm{O}_{3}$ -

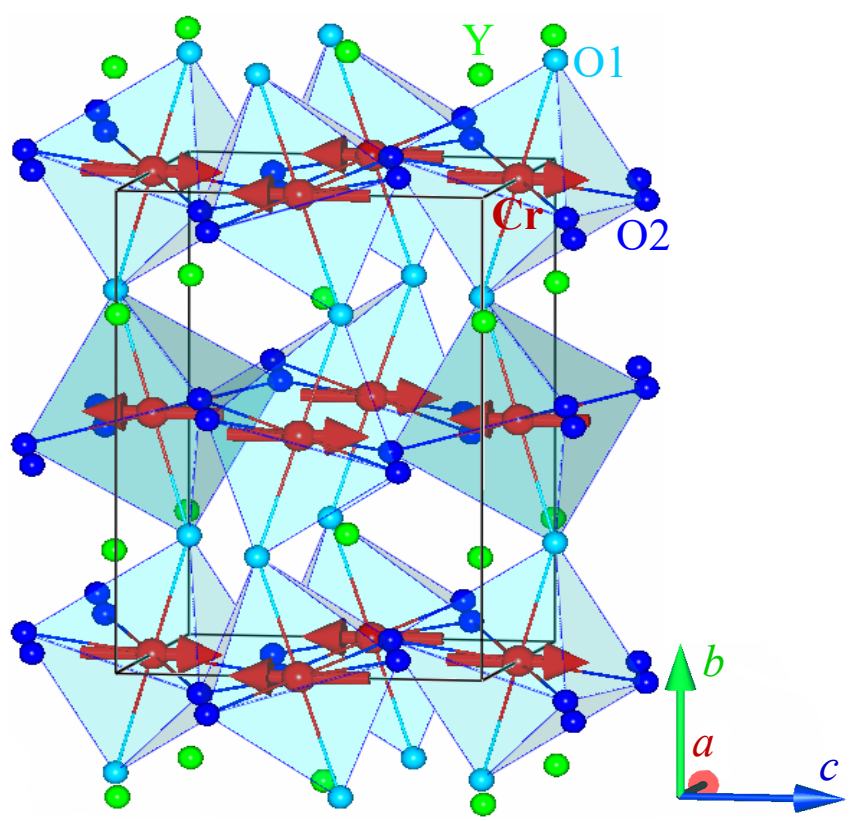

FIG. 1. Orthorhombic crystal structure (with space group Pnma) with one unit cell (solid lines) and the AFM structure in one AFM unit cell with the propagation vector at $\mathbf{k}=(1$ $10)$ below $T_{\mathrm{N}}=141.5(1) \mathrm{K}$ of the $\mathrm{YCrO}_{3}$ single crystal. The arrows on the $\mathrm{Cr}$ ions represent the spins of chromium. Both the unit cells of orthorhombic and AFM structures are $(a b$ $c)$.

KF flux in a platinum crucible [34 36].

In this paper, we have synthesized a centimeter-sized $\mathrm{YCrO}_{3}$ single crystal with a laser diode floating-zone (FZ) furnace 38 and performed measurements of the chemical compositions, resistivity, and magnetization as functions of temperature and applied-magnetic field, heat capacity, time-of-flight neutron-powder diffraction based on a spallation neutron source, and single-crystal neutron diffraction based on a reactor. The chemical compositions of the grown $\mathrm{YCrO}_{3}$ single crystal are nearly stoichiometric, and the $\mathrm{YCrO}_{3}$ compound is a robust insulator.

\section{EXPERIMENTAL}

The preparation of polycrystalline samples of $\mathrm{YCrO}_{3}$ compound has been described previously in detail [14. The single crystals of $\mathrm{YCrO}_{3}$ compound were grown by the FZ method [13] with a laser diode FZ furnace (Model: LD-FZ-5-200W-VPO-PC-UM) [38, 39]. We employed inductively coupled plasma with optical emission spectroscopy (ICP-OES) analysis to quantitatively determine chemical compositions of the investigated single crystals.

We measured resistivity, magnetization, and heat capacity of the $\mathrm{YCrO}_{3}$ samples with a Quantum Design physical property measurement system (PPMS Dyna- 

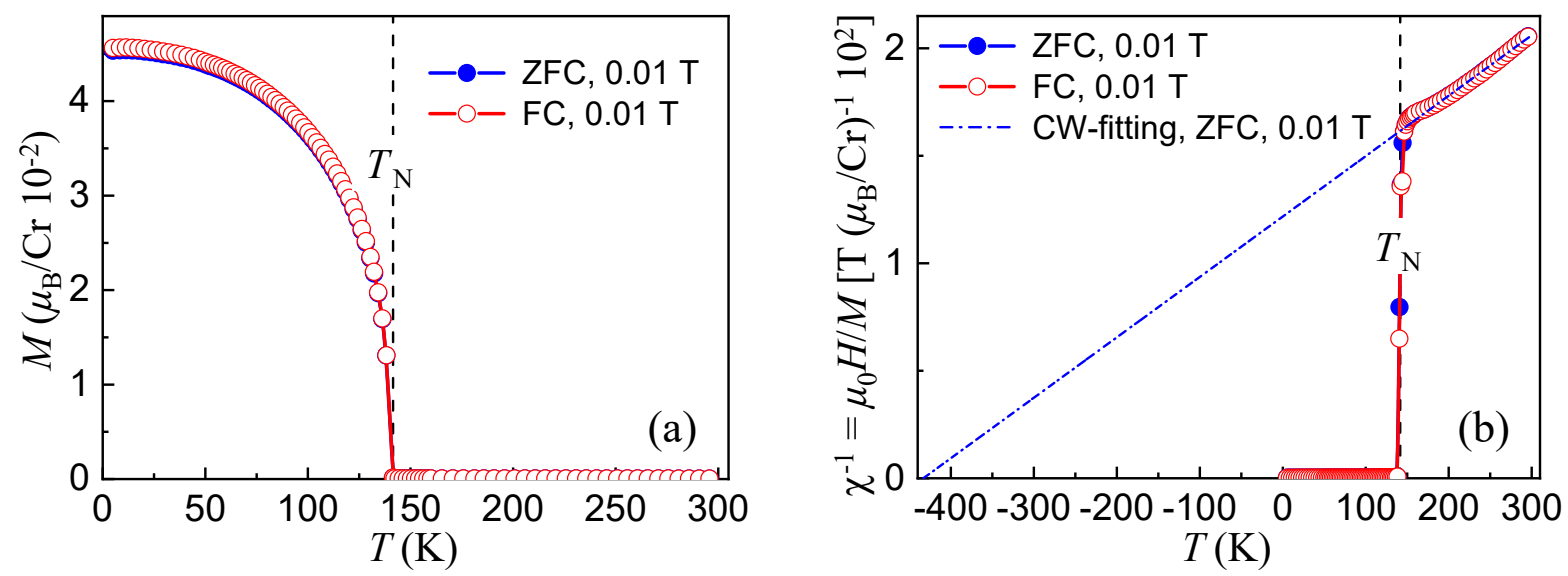

FIG. 2. (a) ZFC and FC magnetization $(M)$ of chromium ions in the single-crystal $\mathrm{YCrO}_{3}$ compound as a function of temperature measured at $\mu_{0} H=0.01 \mathrm{~T}$. (b) Corresponding ZFC and FC inverse magnetic susceptibility $\chi^{-1}$ (circles) of chromium ions in the single-crystal $\mathrm{YCrO}_{3}$ compound versus temperature. The dash-dotted line indicates a $\mathrm{CW}$ behavior of the ZFC data at elevated temperatures between 200 and $300 \mathrm{~K}$, which was extrapolated to $\chi^{-1}=0$ to show the PM Curie temperature $\theta_{\mathrm{CW}}$. The fit results were listed in Table I In (a) and (b), $T_{\mathrm{N}}=141.5(1) \mathrm{K}$ labels the AFM transition temperature at $\mu_{0} H=0.01 \mathrm{~T}$, and the solid lines are guides to the eye.

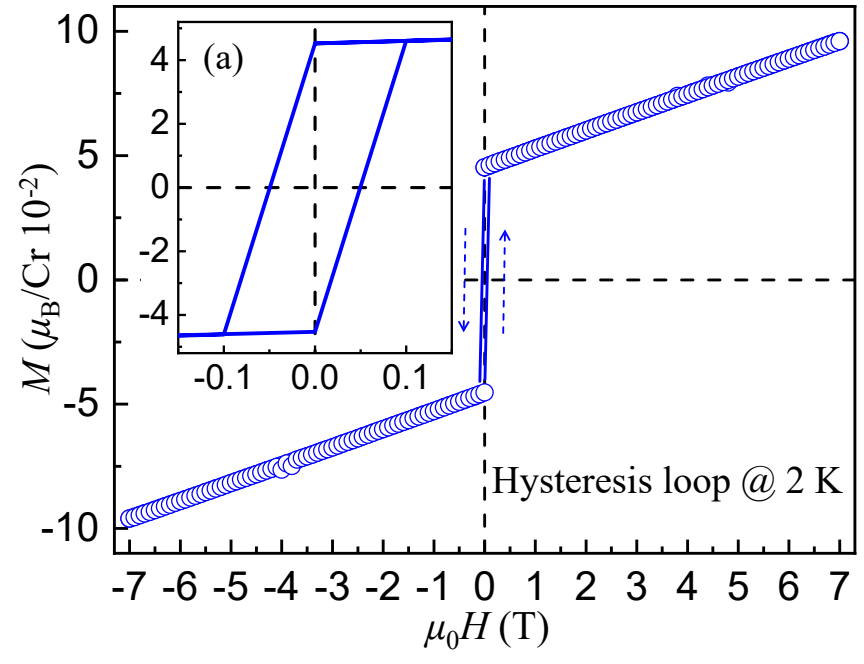

FIG. 3. ZFC magnetic hysteresis loop of the single-crystal $\mathrm{YCrO}_{3}$ compound measured at $2 \mathrm{~K}$. Inset (a) is the enlarged image of the narrow loop.

Cool instrument). Resistivity measurements were carried out with the standard four-probe method at zero field from 2 to $300 \mathrm{~K}$. The dc magnetization measurements at an applied-magnetic field of $0.01 \mathrm{~T}$ were carried out with two modes from 5 to $295 \mathrm{~K}$ : One was after cooling with zero magnetic field (ZFC), and the other was under the applied-magnetic field (FC). Magnetic hysteresis loop from 7 to $-7 \mathrm{~T}$ and then back to $7 \mathrm{~T}$ was measured at $2 \mathrm{~K}$. Heat capacities were measured at $0(2-273 \mathrm{~K})$ and $5 \mathrm{~T}(2-205 \mathrm{~K})$.

We pulverized one grown $\mathrm{YCrO}_{3}$ single crystal $(\sim 4$ g) with a Vibratory Micro Mill (FRITSCH PULVERISETTE 0) and performed a time-of-flight neutron-
TABLE I. Theoretical quantum numbers of $\mathrm{YCrO}_{3}$ compound: spin $S$, orbital $L$, total angular momentum $J$, as well as the ground-state term ${ }^{2 S+1} L_{J}$. Due to a quenching by the hosted crystal field, the actual orbital angular momentum $L$ $=0$ for the $3 d$ ions in most cases, leading to the Landé factor $g_{J}=2$. We also summarized the theoretical (theo.) and measured (meas.) [Fig. 2(b)] values of effective (eff) chromium moment $\mu_{\text {eff }}$, PM Curie temperature $\theta_{\mathrm{CW}}$, theoretical saturation (sat) chromium moment $\mu_{\text {sat_theo., and AFM transition }}$ Néel temperatures $\left(T_{\mathrm{N}}\right)$ at $\mu_{0} H=0.01$ and $5 \mathrm{~T}$. The refined chromium moment size ( $\left.\mu_{\text {meas. }}\right)$ at $12 \mathrm{~K}$ with the AFM model as shown in Fig. 1 from our POWGEN study was listed. The numbers in parenthesis are the estimated standard deviations of the last significant digit.

\begin{tabular}{ll}
\hline \hline \multicolumn{1}{c}{$\mathrm{A} \mathrm{YCrO}_{3}$ single crystal } \\
\hline $3 d$ ion & $\mathrm{Cr}^{3+}$ \\
$3 d^{\mathrm{n}}$ & 3 \\
$S$ & $3 / 2$ \\
$L$ & 3 \\
$J=L-S\left(\right.$ Hund's rule for free $\left.\mathrm{Cr}^{3+}\right)$ & $3 / 2$ \\
$2 S+1 L_{J}$ & ${ }^{4} F_{\frac{3}{2}}$ \\
\hline$g_{J}($ quenched $L=0, J=S)$ & 2 \\
$\mu_{\text {eff_theo. }}=g_{J}[S(S+1)]^{\frac{1}{2}}\left(\mu_{\mathrm{B}}\right)$ & 3.873 \\
$\mu_{\text {sat_theo. }}=g_{J} S\left(\mu_{\mathrm{B}}\right)$ & 3 \\
\hline$\mu_{\text {eff_meas. }}\left(\mu_{\mathrm{B}}\right)$ & $3.95(2)$ \\
$\theta_{\mathrm{CW}}(\mathrm{K})$ & $-433.2(6)$ \\
$T_{\mathrm{N}}($ at $0.01 \mathrm{~T})$ & $141.5(1)$ \\
$T_{\mathrm{N}}($ at $5 \mathrm{~T})$ & $144.5(1)$ \\
$\mu_{\text {meas. }}(12 \mathrm{~K}, \mathrm{POWGEN})\left(\mu_{\mathrm{B}}\right)$ & $2.45(6)$ \\
\hline \hline
\end{tabular}

powder diffraction study on the POWGEN diffractometer (SNS, USA) from 12 to $302 \mathrm{~K}$ at $0 \mathrm{~T}$. The $d$ band cov- 


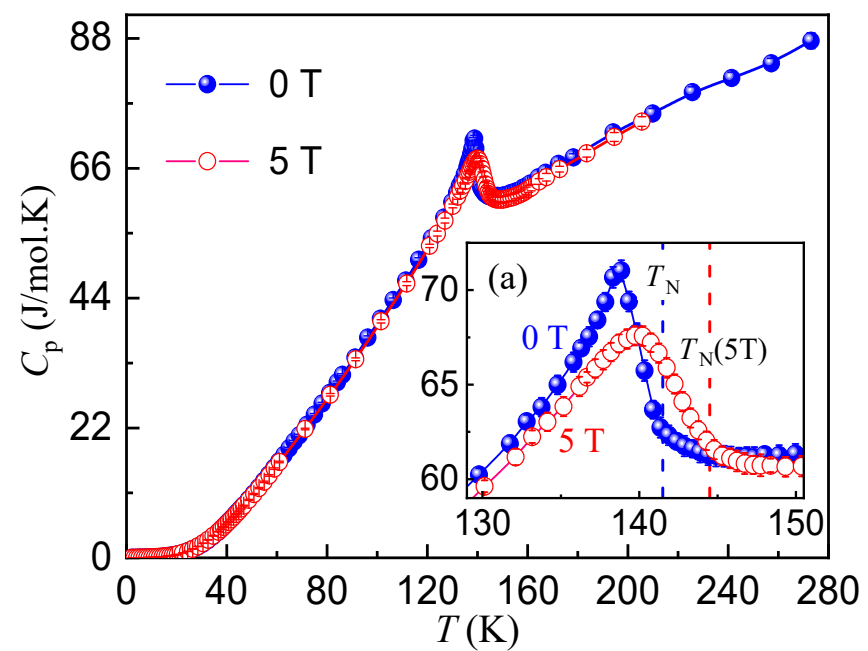

FIG. 4. Heat capacities of the single-crystal $\mathrm{YCrO}_{3}$ compound measured at $0 \mathrm{~T}$ (solid circles) and $5 \mathrm{~T}$ (void circles). The solid lines are guides to the eye. Inset (a) is the enlarged image around the AFM transition temperatures. The vertical dashed lines show the detailed transition temperatures at the fields of 0 and $5 \mathrm{~T}$. Here, $T_{\mathrm{N}}(0 \mathrm{~T})=141.5(1) \mathrm{K}$ at $0 \mathrm{~T}$; by comparison, at $5 \mathrm{~T}, T_{\mathrm{N}}(5 \mathrm{~T})=144.5(1) \mathrm{K}$. The solid lines are guides to the eye.

ers a range of $0.78-7.77 \AA$. Neutron holds a magnetic moment, thus, neutron scattering is a powerful technique for solving magnetic structures [40 43]. The higher $d$ band $(1.7-7.77 \AA)$ is able to monitor all magnetic Bragg reflections that are used to refine the low-temperature magnetic structure. Data from the lower $d$ band (0.78-3.00 $\AA$ ) give indication of all possible structural phase transitions. We analyzed all collected time-of-flight neutronpowder diffraction data with the software of FULLPROF SUITE [44. We refined scale factor, lattice constants, zero epithermal shift, background, peak profile shape, atomic positions, isotropic thermal parameters, and preferred orientation.

Single-crystal neutron diffraction was performed at the D23 diffractometer, located at the Institut Laue-Langevin (ILL), France.

\section{RESULTS AND DISCUSSION}

\section{A. ICP-OES measurements}

By quantitative ICP-OES measurements, we determined the chemical compositions of the studied single crystal as $\mathrm{Y}_{0.97(2)} \mathrm{Cr}_{0.98(2)} \mathrm{O}_{3.00(2)}$. This implies that the resultant single crystals of $\mathrm{YCrO}_{3}$ compound by our FZ method are nearly stoichiometric within the experimental accuracy. Therefore, during analyzing magnetization and time-of-flight neutron-powder diffraction data, we kept the stoichiometry of the synthesized $\mathrm{YCrO}_{3}$ samples be-
TABLE II. Refined structural parameters of the pulverized $\mathrm{YCrO}_{3}$ single crystal at 12,145 , and $300 \mathrm{~K}$, including lattice constants, unit-cell volume, atomic positions, isotropic thermal parameters $(B)$, bond lengths, bond angles, and the distortion parameter $\Delta[14$. We listed the Wyckoff site of each ion and the goodness of fit. The numbers in parenthesis are the estimated standard deviations of the last significant digit.

\begin{tabular}{|c|c|c|c|}
\hline \multicolumn{4}{|c|}{$\begin{array}{c}\text { A pulverized } \mathrm{YCrO}_{3} \text { single crystal } \\
\text { (Orthorhombic, space group Pnma (No. } 62), Z=4)\end{array}$} \\
\hline$T(\mathrm{~K})$ & 12 & 145 & 300 \\
\hline$a(\AA)$ & $5.5189(1)$ & $5.5181(1)$ & $5.5198(1)$ \\
\hline$b(\AA)$ & $7.5205(1)$ & $7.5213(1)$ & $7.5297(1)$ \\
\hline$c(\AA)$ & $5.2323(1)$ & $5.2328(1)$ & $5.2392(1)$ \\
\hline$\alpha(\beta, \gamma)\left(^{\circ}\right)$ & 90 & 90 & 90 \\
\hline$V\left(\AA^{3}\right)$ & $217.17(1)$ & $217.18(1)$ & $217.75(1)$ \\
\hline $\mathrm{Y}(4 c) x$ & $0.0682(4)$ & $0.0672(1)$ & $0.0665(1)$ \\
\hline $\mathrm{Y}(4 c) y$ & 0.25 & 0.25 & 0.25 \\
\hline $\mathrm{Y}(4 c) z$ & $-0.0172(4)$ & $-0.0177(1)$ & $-0.0174(2)$ \\
\hline $\mathrm{Y}(4 c) B\left(\AA^{2}\right)$ & 0.2 & $0.28(2)$ & $0.47(2)$ \\
\hline $\operatorname{Cr}(4 b)(x, y, z)$ & $(0,0,0.5)$ & $(0,0,0.5)$ & $(0,0,0.5)$ \\
\hline $\operatorname{Cr}(4 b) B\left(\AA^{2}\right)$ & 0.2 & $0.29(3)$ & $0.39(3)$ \\
\hline $\mathrm{O} 1(4 c) x$ & $0.4643(5)$ & $0.4646(2)$ & $0.4647(2)$ \\
\hline $\mathrm{O} 1(4 c) y$ & 0.25 & 0.25 & 0.25 \\
\hline $\mathrm{O} 1(4 c) z$ & $0.1039(5)$ & $0.1052(2)$ & $0.1050(2)$ \\
\hline $\mathrm{O} 1(4 c) B\left(\AA^{2}\right)$ & 0.2 & $0.34(2)$ & $0.51(2)$ \\
\hline $\mathrm{O} 2(8 d) x$ & $0.3020(4)$ & $0.3020(1)$ & $0.3021(1)$ \\
\hline $\mathrm{O} 2(8 d) y$ & $0.0539(2)$ & $0.0538(1)$ & $0.0536(1)$ \\
\hline $\mathrm{O} 2(8 d) z$ & $-0.3065(4)$ & $-0.3067(1)$ & $-0.3066(1)$ \\
\hline $\mathrm{O} 2(8 d) B\left(\AA^{2}\right)$ & 0.2 & $0.34(2)$ & $0.51(2)$ \\
\hline Y-O11 $(\AA)$ & $2.237(3)$ & $2.231(1)$ & $2.233(1)$ \\
\hline Y-O12 $(\AA)$ & $2.276(4)$ & $2.286(1)$ & $2.290(1)$ \\
\hline Y-O21 $(\AA)(\times 2)$ & $2.277(3)$ & $2.272(1)$ & $2.273(1)$ \\
\hline Y-O22 $(\AA)(\times 2)$ & $2.476(3)$ & $2.479(1)$ & $2.485(1)$ \\
\hline$<\mathrm{Y}-\mathrm{O}>(\AA)$ & $2.337(1)$ & $2.337(1)$ & $2.340(1)$ \\
\hline Cr-O1 $(\AA)(\times 2)$ & $1.967(1)$ & $1.969(1)$ & $1.971(1)$ \\
\hline $\mathrm{Cr}-\mathrm{O} 21(\AA)(\times 2)$ & $1.983(2)$ & $1.983(1)$ & $1.984(1)$ \\
\hline $\mathrm{Cr}-\mathrm{O} 22(\AA)(\times 2)$ & $1.992(2)$ & 1.991(1) & $1.993(1)$ \\
\hline$<\mathrm{Cr}-\mathrm{O}>(\AA)$ & $1.980(1)$ & $1.981(1)$ & $1.983(1)$ \\
\hline$\angle \mathrm{Cr}-\mathrm{O} 1-\mathrm{Cr}\left({ }^{\circ}\right)$ & $145.81(3)$ & $145.49(1)$ & $145.53(1)$ \\
\hline$\angle \mathrm{Cr}-\mathrm{O} 2-\mathrm{Cr}\left({ }^{\circ}\right)$ & $146.18(9)$ & $146.18(3)$ & $146.22(3)$ \\
\hline$\Delta(\mathrm{Y})\left(\times 10^{-4}\right)$ & 18.197 & 19.085 & 19.789 \\
\hline$\Delta(\mathrm{Cr})\left(\times 10^{-4}\right)$ & 0.268 & 0.215 & 0.203 \\
\hline$\Delta(\mathrm{O} 1)\left(\times 10^{-4}\right)$ & 47.429 & 47.743 & 47.930 \\
\hline$\Delta(\mathrm{O} 2)\left(\times 10^{-4}\right)$ & 90.063 & 90.578 & 91.684 \\
\hline$R_{\mathrm{p}}$ & 6.40 & 5.46 & 6.03 \\
\hline$R_{\mathrm{wp}}$ & 9.33 & 3.67 & 3.74 \\
\hline$R_{\exp }$ & 7.33 & 2.69 & 2.83 \\
\hline$\chi^{2}$ & 1.62 & 1.86 & 1.75 \\
\hline
\end{tabular}

ing the ideal one (i.e., 1:1:3). 


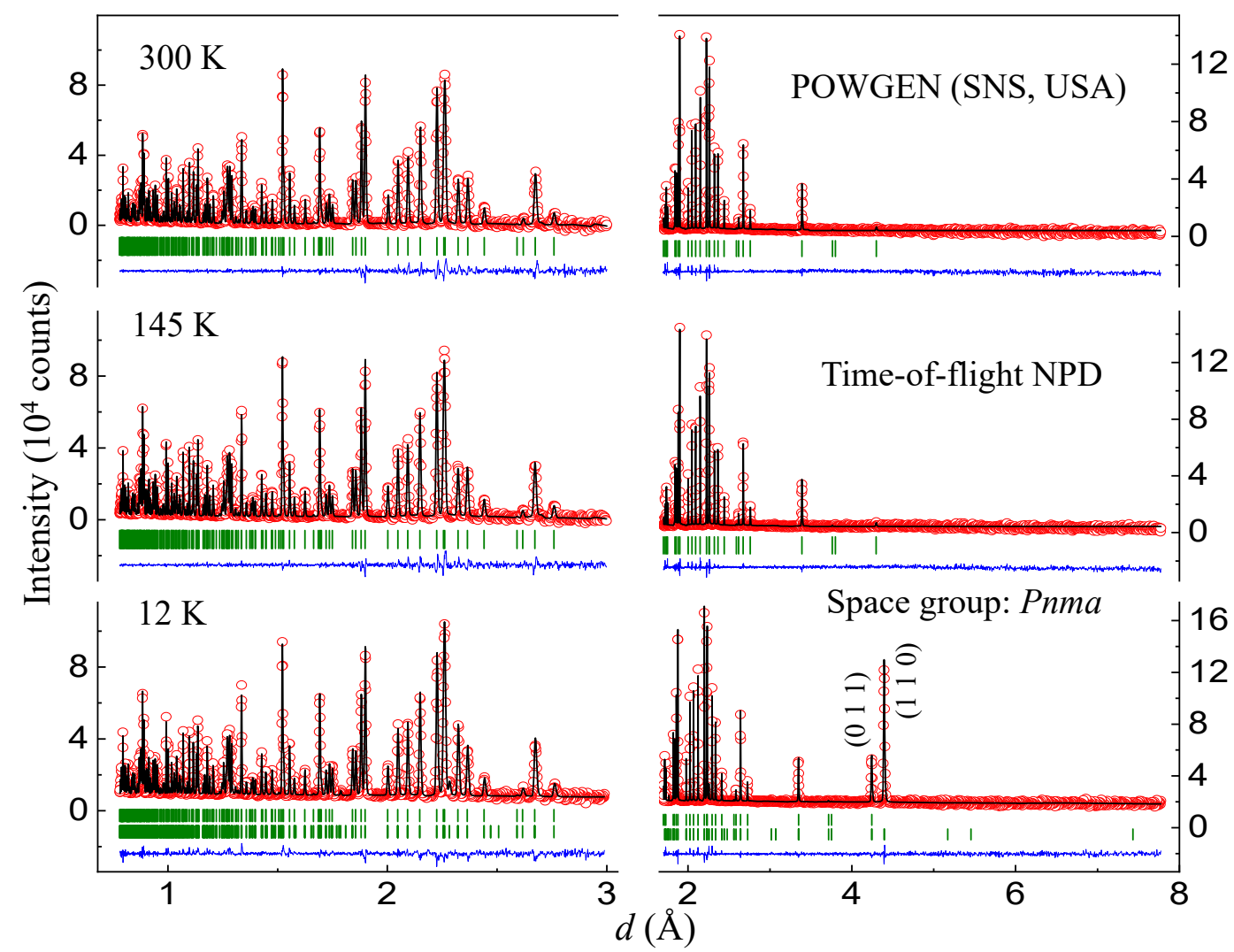

FIG. 5. Observed (circles) and calculated (solid lines) time-of-flight neutron-powder diffraction (NPD) patterns of a pulverized $\mathrm{YCrO}_{3}$ single crystal, collected from the POWGEN diffractometer (SNS, USA) at 12, 145, and $300 \mathrm{~K}$. The vertical bars mark the positions of nuclear (up, space group Pnma) and magnetic (down, space group P-1) Bragg reflections, and the lower curves represent the difference between observed and calculated patterns.

\section{B. Resistivity measurements}

We tried to measure possible resistivity in the $\mathrm{YCrO}_{3}$ single crystals with a multimeter at room temperature. Unfortunately, it was beyond the maximum range $\left(10^{6}\right.$ ohm) of the ohmmeter. In addition, our attempt to measure it by the standard four-probe method with our PPMS DynaCool system from 2 to $300 \mathrm{~K}$ was fruitless. Therefore, we conclude that the $\mathrm{YCrO}_{3}$ compound is a robust insulator in our studied temperature range. A deeper understanding of the electronic states of conducting VO 45 and insulating $\mathrm{YCrO}_{3}$ compounds necessitates more experimental work and theoretical band structure calculations. Perhaps both samples are the only two pure $3 d^{3}$ compounds.

\section{Magnetization versus temperature}

Fig. 2(a) shows magnetization measurements of a small piece of randomly-orientated $\mathrm{YCrO}_{3}$ single crystal. We transferred the unit of vertical axis into $\mu_{\mathrm{B}}$ per $\mathrm{Cr}^{3+}$ ion. There exists no obvious difference between ZFC and FC data. Upon cooling, ZFC and FC magnetiza- tion curves measured at $0.01 \mathrm{~T}$ show very small values down to temperature $\sim 141.5 \mathrm{~K}$, e.g., ZFC magnetization $=4.860(4) \times 10^{-7}$ and $7.310(6) \times 10^{-7} \mu_{\mathrm{B}} / \mathrm{Cr}^{3+}$ at 295 and $142.3 \mathrm{~K}$, respectively. Around $141.5 \mathrm{~K}$, they increase sharply by $\sim 38 \%$ in a small thermal range of $\sim 5 \mathrm{~K}$, followed by a smooth increase down to $5 \mathrm{~K}$. This resembles the characteristic feature of a reasonable canted antiferromagnet and rules out the possibility for a ferrimagnet. At $5 \mathrm{~K}$, ZFC magnetization $=4.530(4) \times 10^{-2} \mu_{\mathrm{B}} / \mathrm{Cr}^{3+}$.

We calculated the inverse magnetic susceptibility $\chi^{-1}=\mu_{0} H / M$ as shown in Fig. 2(b), where the nearly linear increase of $\chi^{-1}$ in the PM state at high temperatures obeys well the molar susceptibility via a CW law

$$
\chi(T)=\frac{C}{T-\theta_{\mathrm{CW}}}=\frac{N_{A} \mu_{\mathrm{eff}}^{2}}{3 k_{B}\left(T-\theta_{\mathrm{CW}}\right)},
$$

where $C$ is the Curie constant, $\theta_{\mathrm{CW}}$ is the PM Curie temperature, $N_{A}=6.022 \times 10^{23} \mathrm{~mol}^{-1}$ is the Avogadro's number, $\mu_{\mathrm{eff}}=g \mu_{\mathrm{B}} \sqrt{J(J+1)}$ is the effective PM moment, and $k_{B}=1.38062 \times 10^{-23} \mathrm{~J} / \mathrm{K}$ is the Boltzmann constant. The fit by Eq. (1) was shown as the dashdotted line in Fig. 2(b). The fit parameters were listed in Table 1

Figures 2(a) and 2(b) clearly indicate a sharp mag- 


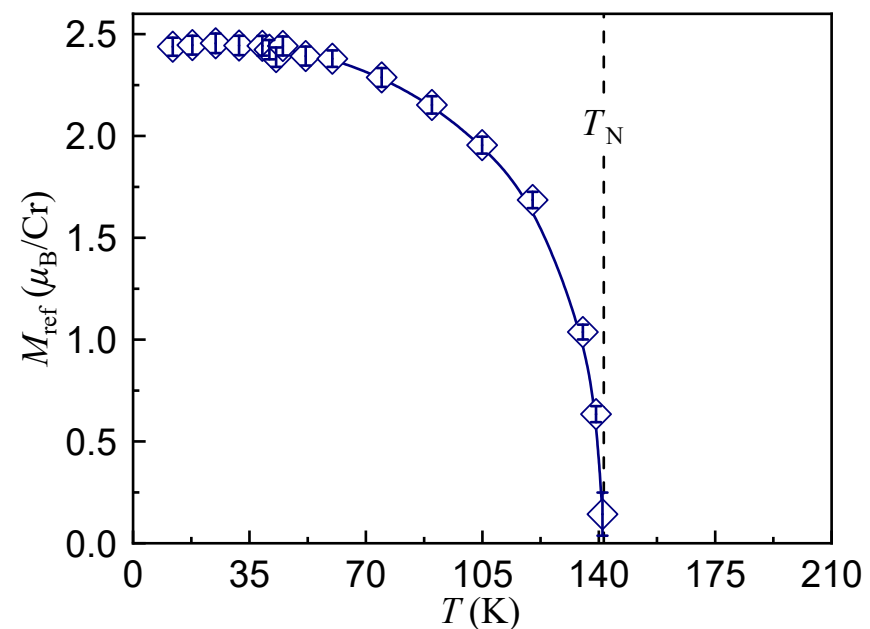

FIG. 6. Refined chromium-moment size $M_{\text {ref }}($ at $0 \mathrm{~T}$ ) of a pulverized $\mathrm{YCrO}_{3}$ single crystal versus temperature by the software of FULLPROF SUITE [44. The solid line is a guide to the eye. Error bars are standard deviations obtained from our FULLPROF refinements in the Pnma symmetry. $T_{\mathrm{N}}=$ 141.5(1) K labels the AFM transition temperature at zero applied-magnetic field.

netic phase transition. We determined the magnetic phase transition temperature as $T_{\mathrm{N}}=141.5(1) \mathrm{K}$. The resultant $\mathrm{PM} \mathrm{CW}$ temperature $\theta_{\mathrm{CW}}=-433.2(6) \mathrm{K}$, indicating an existence of strong AFM correlations. We calculated the frustrating parameter [46-51, i.e., $f=1$ $\theta_{\mathrm{CW}} \mid / T_{\mathrm{N}}=3.061(5)$, which was consistent with our high-temperature magnetization study [14. This value indicates that the low-temperature magnetic moments of $\mathrm{Cr}^{3+}$ ions in $\mathrm{YCrO}_{3}$ compound are frustrated by competitive spin exchanges. As listed in Table I the extracted effective PM moment $\mu_{\text {eff_meas }}=3.95(2) \mu_{\mathrm{B}}$, a little bit larger than the calculated theoretical value $\mu_{\text {eff_theo }}=$ $3.873 \mu_{\mathrm{B}}$, which was acceptable within the present experimental accuracy.

\section{Magnetization versus applied magnetic field}

Figure 3 shows the measurement of magnetic hysteresis loop at 2 K. Figure 3(a) clearly exhibits the hysteresis loop whose shape is of a parallelogram. The loop locates in a magnetic field range of $\sim-0.1$ to $0.1 \mathrm{~T}$ with a coersive force of $\sim 0.05 \mathrm{~T}$ and a residual magnetism of $\sim 4.54$ $\times 10^{-2} \mu_{\mathrm{B}} / \mathrm{Cr}^{3+}$. These small values demonstrate that the $\mathrm{YCrO}_{3}$ compound is a soft canted antiferromagnet at low temperatures. At $2 \mathrm{~K}$ and $7 \mathrm{~T}$, the measured ZFC magnetization $M=9.603 \times 10^{-2} \mu_{\mathrm{B}} / \mathrm{Cr}^{3+}$. From 0.1 to 7 $\mathrm{T}$, the measured magnetization almost increases linearly with $\chi=M / \mu_{0} H=7.24(1) \times 10^{-3} \mu_{\mathrm{B}} \mathrm{T}^{-1} / \mathrm{Cr}^{3+}$. We therefore estimated that reaching a complete magnetic saturation state, an applied-magnetic field $\mu_{0} H \geq \sim 408$ $\mathrm{T}$ is required 52 .

\section{E. Heat capacity}

Figure 4 shows the heat capacity measurements. At 0 $\mathrm{T}$, with decreasing temperature, the measured heat capacity decreases until $T_{\mathrm{N}}=141.5 \mathrm{~K}$, followed by an appearance of a $\lambda$-shape peak. Below this, heat capacity continues to decrease and gets flat below $\sim 24 \mathrm{~K}$. The observation of the $\lambda$-shape peak indicates a phase transition. To reveal the nature of the phase transition, we measured heat capacity under an applied-magnetic field of $5 \mathrm{~T}$. As shown in Fig. 4 (a), at $5 \mathrm{~T}$, it was noted that the intensity of the $\lambda$-shape peak was reduced, accompanied by a shift of the peak position from $\sim 138.8 \mathrm{~K}(0 \mathrm{~T})$ to an elevated temperature $\sim 139.9 \mathrm{~K}(5 \mathrm{~T})$. This is the characteristic feature of a canted antiferromagnet. Thus, the phase transition is magnetic rather than structural. We determined $T_{\mathrm{N}}(5 \mathrm{~T})=144.5(1) \mathrm{K}, \sim 3 \mathrm{~K}$ higher than the $T_{\mathrm{N}}$ at $0 \mathrm{~T}$. Quantitative analysis of the relationship between values of $T_{\mathrm{N}}$ and applied-magnetic-field strengths necessitates more measurements. It is pointed out that with the measurement of magnetization versus temperature, it is easy to determine the value of $T_{\mathrm{N}}$. From heat capacity measurements, the phase transition temperature is at the temperature point at which a kink exists in the $C_{\mathrm{p}}-T$ curve as marked in Fig. 4(a).

Albeit that the magnetization and heat capacity measurements show FM behaviors below $T_{\mathrm{N}}$, the net magnetic interaction strength inside $\mathrm{YCrO}_{3}$ compound is of strongly AFM because $\theta_{\mathrm{CW}}=-433.2(6) \mathrm{K}$, indicating a complex low-temperature magnetic structure.

\section{F. Time of flight neutron powder diffraction}

To make the nature of the observed weak ferromagnetism clear and explore possible structural phase transitions in the $\mathrm{YCrO}_{3}$ single crystal, we performed a timeof-flight neutron-powder diffraction study. The results were shown in Fig. 5. At the three temperatures as labeled, i.e., below $(12 \mathrm{~K})$, around $(145 \mathrm{~K})$, and above (300 $\mathrm{K})$ the magnetic transition temperature $(\sim 141.5 \mathrm{~K})$, all time-of-flight neutron-powder diffraction patterns were well indexed by an orthorhombic structure with the space group Pnma. There was no detectable peak splitting or an appearance of satellite reflections. This indicates that no structural phase transition occurs in the $\mathrm{YCrO}_{3}$ single crystal as a function of temperature in the studied thermal regime. This is in agreement with our heat capacity measurements. Based on the observed magnetic Bragg $\left(\begin{array}{lll}0 & 1 & 1\end{array}\right)$ and $\left(\begin{array}{lll}1 & 1 & 0\end{array}\right)$ peaks, as labeled in the right-bottom of Fig. 5, we established an AFM model with the propagation vector at $\mathbf{k}=\left(\begin{array}{lll}1 & 1 & 0\end{array}\right)$ and the moment directions along the crystallographic $c$ axis. The extracted magnetic structure was schematically drawn in Fig. 1. It is pointed out that the magnetic ( $\left.\begin{array}{lll}1 & 1 & 0\end{array}\right)$ reflection is structurally forbidden by the space group Pnma. We tried 

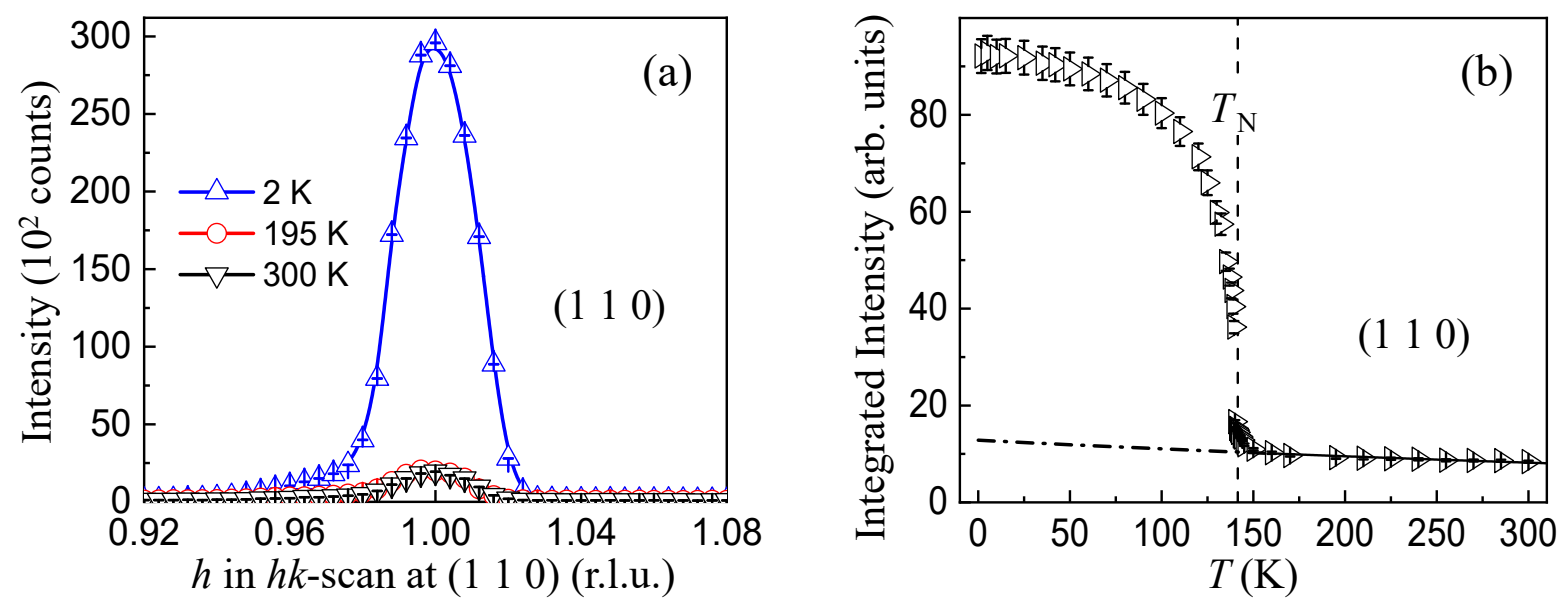

FIG. 7. (a) Representative longitudinal scans of the magnetic Bragg (1 10 ) reflection at three temperatures of 2, 195, and $300 \mathrm{~K}$, from the D23 (ILL, France) study on a $\mathrm{YCrO}_{3}$ single crystal. The solid lines are guides to the eye. (b) Corresponding temperature-dependent integrated intensities of the magnetic Bragg ( 110 l 10 reflection. $T_{\mathrm{N}}=141.5(1) \mathrm{K}$ labels the AFM transition temperature. The solid line was a fit to Eqs. (2) and (4) in the affiliated thermal regime. It was extrapolated to overall temperatures (dash-dotted line). The error bars in (a) and (b) are the standard deviations based on our measurements and fits.

all possible canted AFM models, unfortunately, the corresponding FULLPROF refinements were not successful. The refined structural parameters were listed in Table II. It is pointed out that for the refinement of the data at 12 $\mathrm{K}$, we constrained the isotropic thermal parameters $(B)$ of $\mathrm{Y}, \mathrm{Cr}, \mathrm{O} 1$, and $\mathrm{O} 2$ ions being the same as 0.2 .

Within the present experimental accuracy, we can only determine a G-type AFM structure as shown in Fig. 1. where the nearest-neighbor $\mathrm{Cr}^{3+}$ spins are aligned antiparallel. The directions of the AFM submoments are along with the crystallographic $c$ axis, i.e., the direction with the smallest lattice constant. It is hard to determine the possible canting angle. It was suggested that including a spin-orbital coupling on the quenched $\mathrm{Cr}^{3+}$ ground state, an antisymmetric exchange interaction would cant the AFM moments along the crystallographic $b$ axis, i.e., the direction with the largest lattice constant, according to the Dzialoshinski-Moriya theory [53]. This leads to an appearance of the weak ferromagnetism.

Figure 6 shows the refined moment size of $\mathrm{Cr}^{3+}$ ions in the $\mathrm{YCrO}_{3}$ single crystal, extracted from our timeof-flight neutron-powder diffraction study. As listed in Table I, the refined moment size at $12 \mathrm{~K}$ is $2.45(6) \mu_{\mathrm{B}}$, $\sim 82 \%$ of the theoretical saturation moment $\left(3 \mu_{\mathrm{B}}\right)$, in agreement with our conclusion that there exists a magnetic frustration in $\mathrm{YCrO}_{3}$ compound and the studies with $\mathrm{x}$-ray magnetic circular dichroism and absorption spectroscopies [30] where the computed values of spin and orbital moments are $2.38 \mu_{\mathrm{B}}$ and $-0.094 \mu_{\mathrm{B}}$, respectively, and that the total magnetic moment has little contribution from the orbital component. As temperature increases, the refined moment size remains a plateau up to $\sim 50 \mathrm{~K}$, followed by a gradual diminution with tempera-

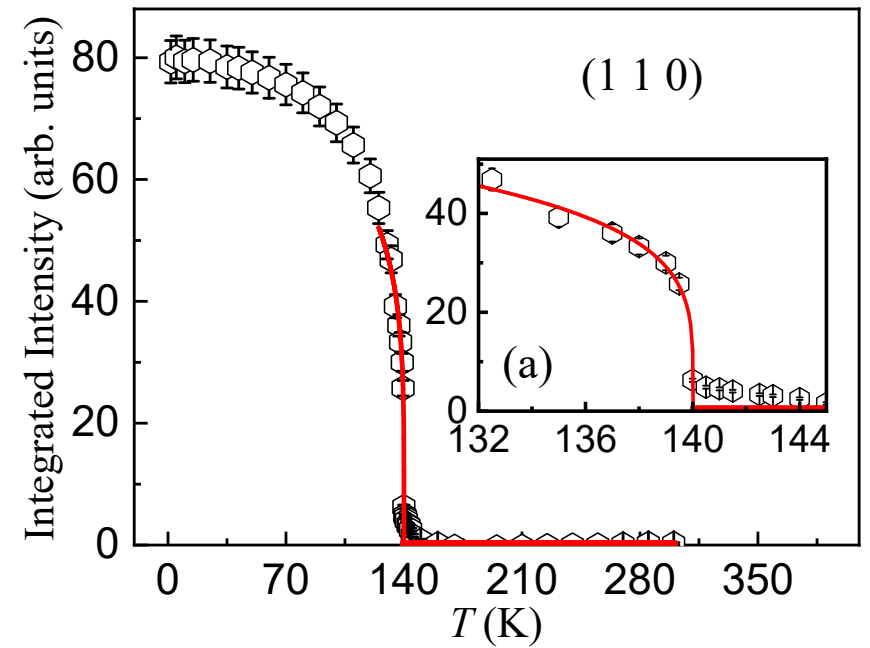

FIG. 8. Subtracted integrated intensity from the pure magnetic contribution at the Bragg (1 10 l $)$ ) peak position (void pentagons), to see detailed analysis in the text. Inset (a) shows the enlarged image around $T_{\mathrm{N}}$ from $132-145 \mathrm{~K}$. The solid line was a fit to the power-law Eq. (5) in the affiliated thermal regime. The error bars are the propagated standard deviations based on our calculations.

ture upon warming until a radical disappearance around 141.5 K, the onset temperature of the AFM transition (Figs. 2 and 6). Above $T_{\mathrm{N}}(0 \mathrm{~T})=141.5 \mathrm{~K}$, intensities of the two magnetic Bragg reflections were undetectable. 


\section{G. Single crystal neutron diffraction}

An orientated $\mathrm{YCrO}_{3}$ single crystal with the $\left(\begin{array}{lll}H & K & 0\end{array}\right)$ reciprocal-lattice vector in the scattering plane was used to perform a single-crystal neutron diffraction study on the D23 single-crystal diffractometer (ILL, France). Figure 7(a) shows some longitudinal scans of the magnetic Bragg ( $\left.\begin{array}{lll}1 & 1 & 0\end{array}\right)$ reflection. At $2 \mathrm{~K}$ (below $T_{\mathrm{N}}$ ), we observed a very strong peak, indicating a formation of the AFM structure. At 195 and $300 \mathrm{~K}$ (above $T_{\mathrm{N}}$ ), the intensity of the magnetic $\left(\begin{array}{lll}1 & 1 & 0\end{array}\right)$ peak decreased sharply but did not disappear. It is interesting that there still exists detectable intensity of the Bragg (1 110$)$ peak above $T_{\mathrm{N}}$. We ruled out the $\lambda / 2$ contamination. As the foregoing remark, this reflection is forbidden by the space group Pnma. Therefore, the existence of the Bragg (1 $10)$ reflection above $T_{\mathrm{N}}$ indicates that the actual crystalline structure of $\mathrm{YCrO}_{3}$ compound may be lower than the orthorhombic structure with space group Pnma. Our studies also demonstrate that the scattering ability of a single crystal is much higher than that of the corresponding pulverized powder sample.

At 195 and $300 \mathrm{~K}$, the observed Bragg ( $\left.\begin{array}{lll}1 & 1 & 0\end{array}\right)$ reflection forbidden structurally by the Pnma symmetry was treated to be from a pure nuclear contribution. The temperature variation of this contribution depends mainly on the thermal dynamic vibrations of related atoms, i.e., Debye-Waller (DW) factors. The falloff of the temperature-weakened intensity at a certain scattering vector $\boldsymbol{Q}$ almost decays exponentially and can be estimated by

$$
I=I_{0} e^{-2 W(Q, T)},
$$

where the exponential part is the DW factor, and

$$
2 W(Q, T)=\frac{\hbar^{2} Q^{2}}{2 \mathrm{M}} \int \operatorname{coth}\left(\frac{\hbar \omega}{2 k_{B} T}\right) \frac{Z(\omega)}{\omega} d \omega,
$$

where $\hbar=1.054589 \times 10^{-34} \mathrm{~J}$.s is the Planck constant divided by $2 \pi, \mathrm{M}$ is the atomic mass, and $Z(\omega)$ is the phonon density of states [54]. At high temperatures, it is given simply by

$$
2 W=\frac{3 \hbar^{2} Q^{2}}{\mathrm{M} k_{B} \Theta_{W}^{2}} T
$$

where $\Theta_{W}$ is the effective Debye temperature [55]. Therefore, we treated practically the DW factor with a linear response to temperature, as verified in previous studies [56 59]. We fit the integrated intensities of the Bragg ( $\left.\begin{array}{lll}1 & 1 & 0\end{array}\right)$ reflection above $T_{\mathrm{N}}$ to Eqs. (2) and (4), shown as the solid line in Fig. 7(b), and extrapolated the fit to the entire temperature range (shown as the dash-dotted line).

Furthermore, we subtracted the corresponding nuclear component from the total scattered intensity at the Bragg (1 110$)$ peak position to extract the pure magnetic

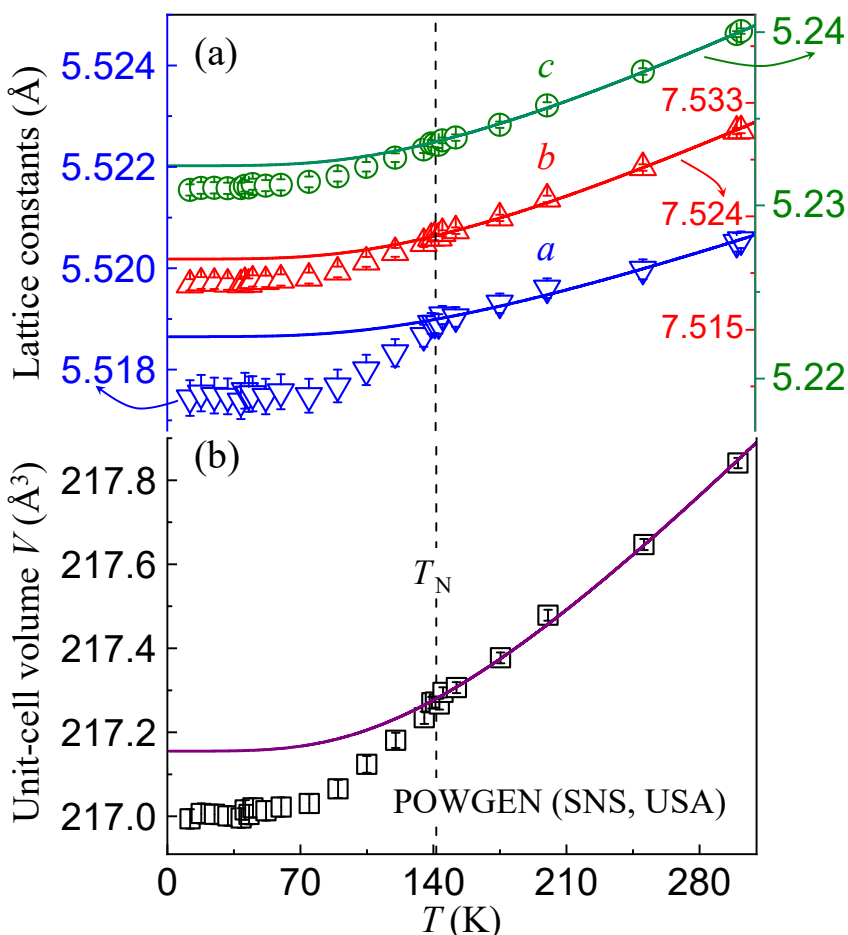

FIG. 9. (a) Temperature-dependent lattice constants $a, b$, and $c$ of a pulverized $\mathrm{YCrO}_{3}$ single crystal. (b) Corresponding anomalous unit-cell volume $V$ expansion with temperature. The solid lines in (a) and (b) are theoretical estimates of the variation of structural parameters using the Grüneisen model with Debye temperature of $\theta_{\mathrm{D}}=580 \mathrm{~K}$ that is the same value as the one reported previously $14 . T_{\mathrm{N}}=141.5(1) \mathrm{K}$ labels the AFM transition temperature. The error bars in (a) and (b) are the standard deviations obtained from our FULLPROF refinements with the Pnma structural symmetry.

contribution below $T_{\mathrm{N}}$ as shown in Fig. 8 . The resultant magnetic intensity above $T_{\mathrm{N}}$ is approximately zero within accuracy, which in turn supports the above subtraction. The extracted integrated intensity $(I)$ of Bragg $\left(\begin{array}{lll}1 & 1 & 0\end{array}\right)$ reflection from the pure magnetic contribution can be fit to a power-law equation 60, 61.

$$
I(T)=I_{0}\left(\frac{\left|T-T_{\mathrm{N}}\right|}{T_{\mathrm{N}}}\right)^{2 \beta},
$$

where $T_{\mathrm{N}}$ is the value of the Néel temperature, and $\beta$ is the critical exponent. Our fit with Eq. (5) to the extracted data in a narrow thermal range from 125 to 140 $\mathrm{K}$, shown as the solid line in Fig. 8, produces a Néel temperature $T_{\mathrm{N} 1}=140.0(1) \mathrm{K}$, and the critical exponent $2 \beta=0.215(6)$, indicating a second-order type phase transition and probably two-dimensional Ising-like spin interactions existing within the reciprocal $\left(\begin{array}{lll}1 & 1 & 0\end{array}\right)$ scattering plane [41, 60. The fitting procedure was as follow: First, we kept $I_{0}=80$ and $T_{\mathrm{N}}=141.5 \mathrm{~K}$ and allowed $\beta$ to vary; finally, we fit all parameters together. For comparison, we further fit the data in three temperature ranges of 125-140 K, 130-140 K, and 135-140 K. No clear differ- 


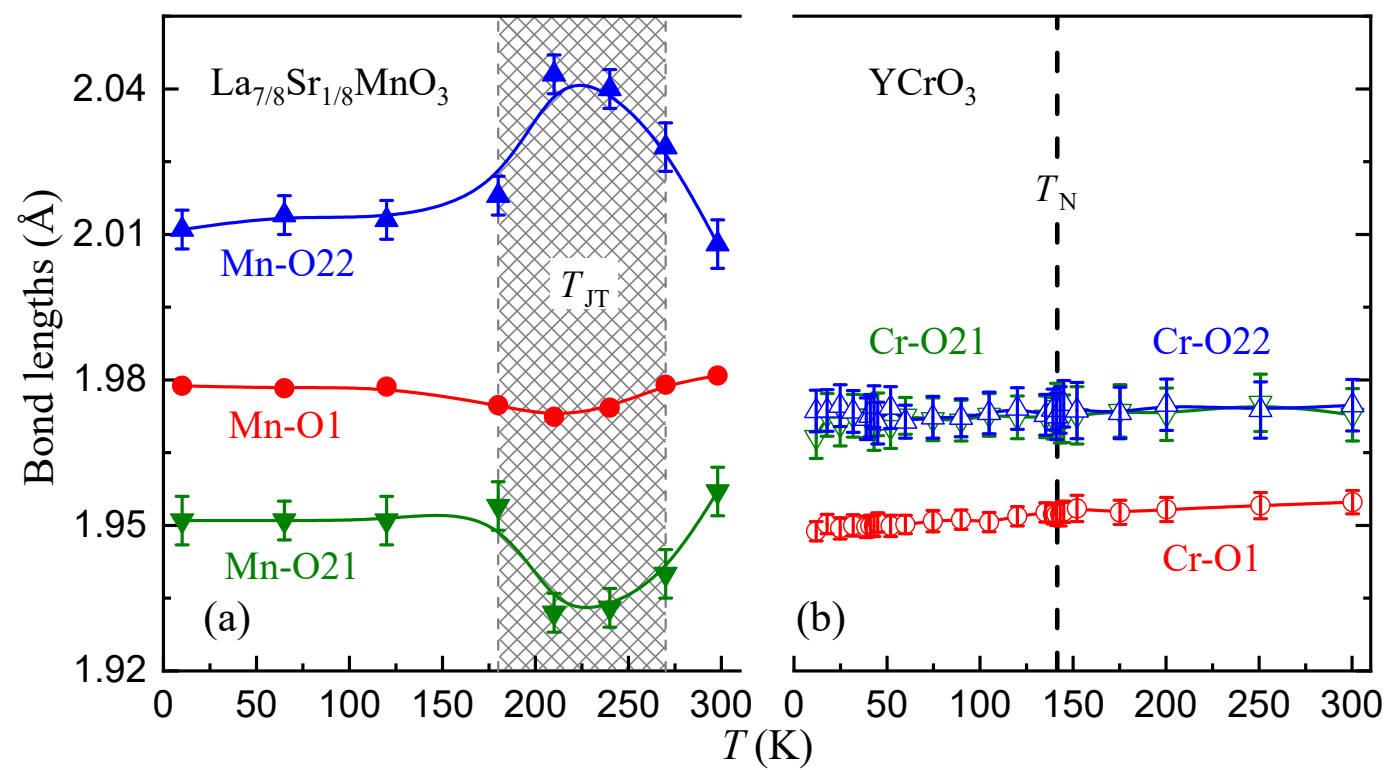

FIG. 10. (a) Three bond lengths of Mn-O1, Mn-O21, and Mn-O22 in the $\mathrm{La}_{\frac{7}{8}} \mathrm{Sr}_{\frac{1}{8}} \mathrm{MnO}_{3}$ single crystal [0] versus temperature. $T_{\text {JT }} \approx 180-270 \mathrm{~K}$ denotes the regime of the Jahn-Teller effect. (b) Corresponding bond lengths in the $\mathrm{YCrO}_{3}$ single crystal as a function of temperature from this study. $T_{\mathrm{N}}=141.5(1) \mathrm{K}$ labels the AFM transition temperature. The error bars in (a) and (b) were from our FULLPROF refinements.

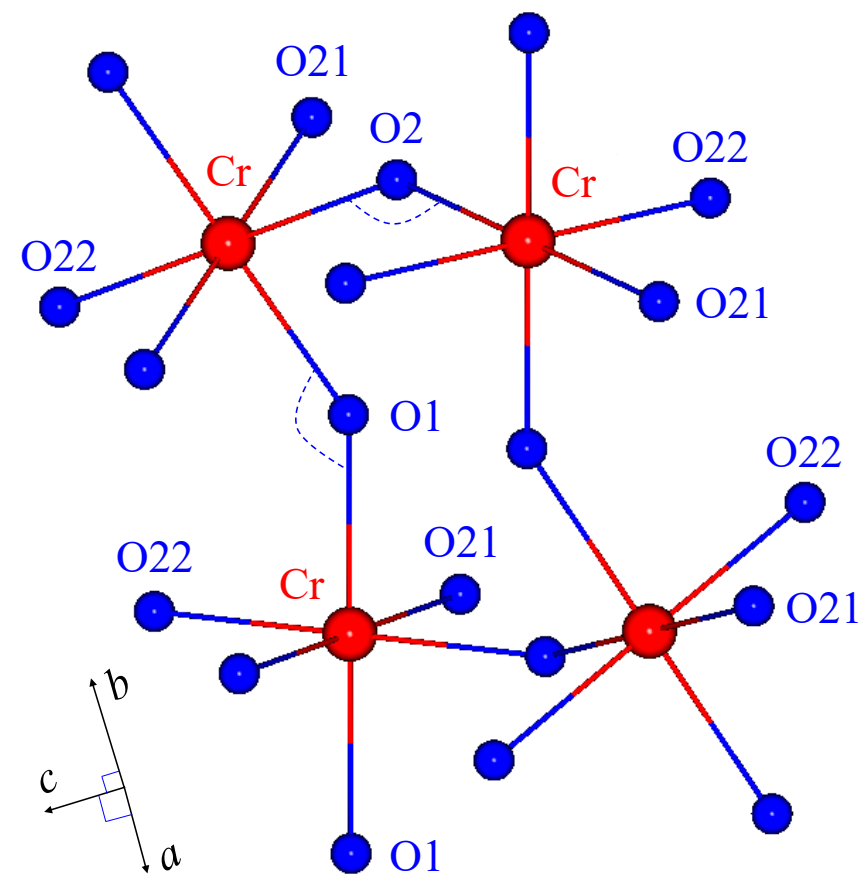

FIG. 11. Schematic illustration of the three Cr-O bonds (CrO1, Cr-O21, and Cr-O22), as well as the two bond angles Cr$\mathrm{O}-\mathrm{Cr}$ (Cr-O1-Cr and Cr-O2-Cr) in the orthorhombic structure of a $\mathrm{YCrO}_{3}$ single crystal. In this structural symmetry (with space group Pnma), $\mathrm{Cr}$ ions in $\mathrm{YCrO}_{3}$ compound have the same Wyckoff site, $4 b$ ( $\left.\begin{array}{lll}0 & 0 & 0.5\end{array}\right)$, as that of the Mn ions in $\mathrm{La}_{\frac{7}{8}} \mathrm{Sr}_{\frac{1}{8}} \mathrm{MnO}_{3}$ compound [13, 40, 56, 57. ences exist in the values of the refined $T_{\mathrm{N}}$ and $\beta$, which validates our choice of the temperature range of $125-140$ $\mathrm{K}$ for the final fitting.

As shown in Fig. 8(a), it is interesting to note that above $T_{\mathrm{N} 1}$, there exists weaker critical scattering over a range of temperature up to $145 \mathrm{~K}$.

\section{H. Anisotropic magnetostriction effect}

The refined lattice parameters $a, b$, and $c$, as well as the unit-cell volume $V$, from our time-of-flight neutronpowder diffraction studies were shown in Fig. 9 (void symbols). Upon cooling, the refined (Re) $a, b, c$, and $V$ almost shrink linearly down to $T_{\mathrm{N}}$ at which a cusp appears.

As in the foregoing discussions, the $\mathrm{YCrO}_{3}$ compound is an insulator. We thus neglected the electronic contribution to the thermal expansion of its lattice configuration $(\varepsilon)$. The temperature variation of the nonmagnetic contribution component is then mainly from phonons. This can approximately be estimated according to the Grüneisen rules at zero pressure with a second-order fashion 62 64]

$$
\varepsilon(T)=\varepsilon_{0}+\varepsilon_{0} \frac{U}{Q-B U},
$$

where $\varepsilon_{0}$ is the lattice parameter at zero Kelvin, and the internal energy $U$ can be calculated with the Debye 


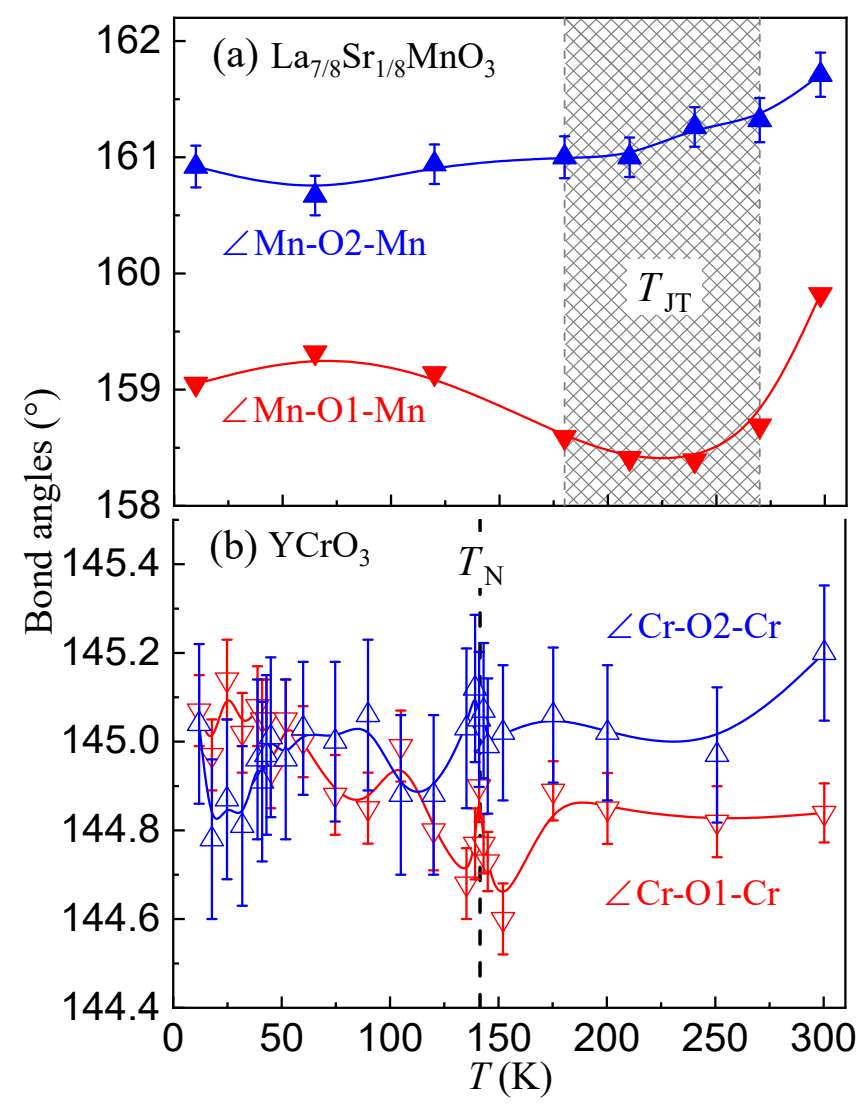

FIG. 12. (a) Temperature-dependent bond angles of Mn-O1$\mathrm{Mn}$ and $\mathrm{Mn}-\mathrm{O} 2-\mathrm{Mn}$ in the $\mathrm{La}_{\frac{7}{8}} \mathrm{Sr}_{\frac{1}{8}} \mathrm{MnO}_{3}$ single crystal [40]. $T_{\mathrm{JT}} \approx 180-270 \mathrm{~K}$ denotes the regime of the Jahn-Teller effect. (b) Temperature-dependent bond angles of Cr-O1-Cr and Cr$\mathrm{O} 2-\mathrm{Cr}$ in the $\mathrm{YCrO}_{3}$ single crystal from the present study. $T_{\mathrm{N}}=141.5(1) \mathrm{K}$ labels the AFM transition temperature. The error bars in (a) and (b) are the standard deviations from refinements. The solid lines in (a) and (b) are guides to the eye.

approximations

$$
U(T)=9 N k_{B} T\left(\frac{T}{\Theta_{D}}\right)^{3} \int_{0}^{\frac{\Theta_{D}}{T}} \frac{x^{3}}{e^{x}-1} d x
$$

where $N=5$ is the number of atoms per formula unit, and $\Theta_{D}$ is the Debye temperature. With Eqs. (6) and (7), we fit the lattice parameters of $\mathrm{YCrO}_{3}$ compound in the PM state (above $T_{\mathrm{N}} \approx 141.5 \mathrm{~K}$ ) and extrapolated the fits to overall temperatures as shown in Fig. 9 (solid lines). For example, the fitting for the unit-cell volume $V$ results in $V_{0} \approx 217.14 \AA^{3}, Q \approx 7.57 \times 10^{-18} \mathrm{~J}$, and $B \approx-40.73$. The different variations in $a, b$, and $c$ below $T_{\mathrm{N}}$ in contrast to our theoretical estimates by the Grüneisen (Gr) law 63, 64, (solid lines), e.g., $\frac{a_{\mathrm{Re}}^{12 \mathrm{~K}}-a_{\mathrm{Gr}}^{12 \mathrm{~K}}}{a_{\mathrm{Gr}}^{12 \mathrm{~K}}}$ $\approx-2.73 \times 10^{-4}, \frac{b_{\mathrm{Re}}^{12 \mathrm{~K}}-b_{\mathrm{Gr}}^{12 \mathrm{~K}}}{b_{\mathrm{Gr}}^{12 \mathrm{~K}}} \approx-2.19 \times 10^{-4}$, and $\frac{c_{\mathrm{Re}}^{12 \mathrm{~K}}-c_{\mathrm{Gr}}^{12 \mathrm{~K}}}{c_{\mathrm{Gr}}^{12 \mathrm{~K}}}$ $\approx-2.67 \times 10^{-4}$, indicate an anisotropic magnetostriction effect and that magnetic anisotropy exists in $\mathrm{YCrO}_{3}$ com-

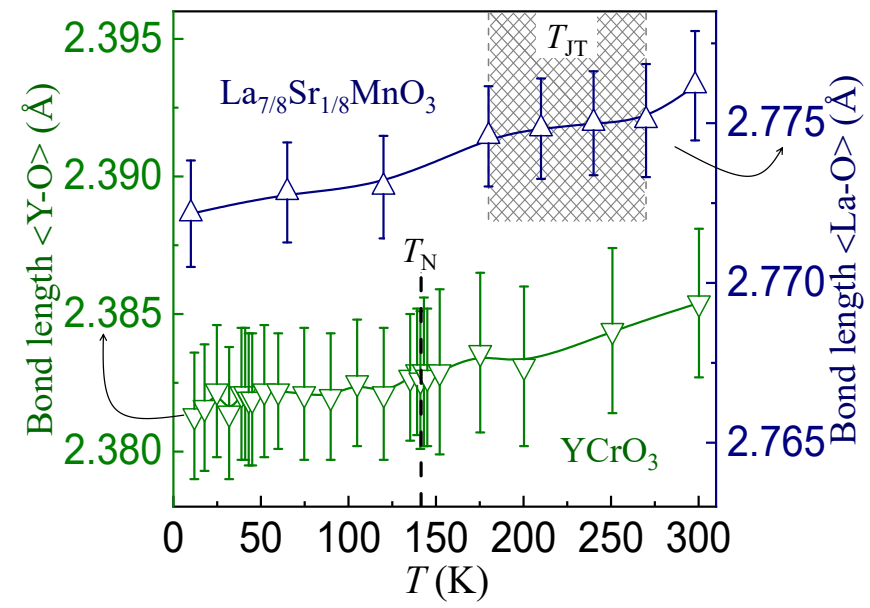

FIG. 13. Comparison of the averaged bond lengths of $\mathrm{Y}-\mathrm{O}$ in $\mathrm{YCrO}_{3}$ (left, from the present study) and La-O in $\mathrm{La}_{\frac{7}{8}} \mathrm{Sr}_{\frac{1}{8}} \mathrm{MnO}_{3}$ [40] (right) single crystals. $T_{\mathrm{JT}} \approx 180-270 \mathrm{~K}$ denotes the regime of the Jahn-Teller effect of $\mathrm{La}_{\frac{7}{8}} \mathrm{Sr}_{\frac{1}{8}} \mathrm{MnO}_{3}$ compound. $T_{\mathrm{N}}=141.5(1) \mathrm{K}$ labels the AFM transition temperature of $\mathrm{YCrO}_{3}$ compound. The error bars are the calculated standard deviations. The solid lines are guides to the eye. It is clear that the bond length of $\langle\mathrm{Y}-\mathrm{O}\rangle$ is shorter than that of the $\langle\mathrm{La}-\mathrm{O}\rangle$ bond beyond statistics.

pound. Below $T_{\mathrm{N}}$, the magnetically-driven additional decreases of $a, b$, and $c$ jointly result in an enhanced sample contraction upon cooling, e.g., $\frac{V_{\mathrm{Re}}^{12 \mathrm{~K}}-V_{\mathrm{Gr}}^{12 \mathrm{~K}}}{V_{\mathrm{Gr}}^{12 \mathrm{~K}}} \approx-7.43 \times$ $10^{-4}$, signifying a magnetoelastic effect [65] and a localized nature of the $t_{2 \mathrm{~g}}$ moments, opposite the case in the GdSi metallic compound 62].

\section{Comparison between $t_{2 \mathrm{~g}} \mathrm{YCrO}_{3}$ and $e_{\mathrm{g}}$ $\mathrm{La}_{7 / 8} \mathrm{Sr}_{1 / 8} \mathrm{MnO}_{3}$ compounds}

As shown in Fig. 10, it is of interest to compare the bond lengths of the $3 d \mathrm{Mn}^{3+} / \mathrm{Mn}^{4+}\left(e_{\mathrm{g}}^{1} t_{2 \mathrm{~g}}^{3} / e_{\mathrm{g}}^{0} t_{2 \mathrm{~g}}^{3}\right)$ ions in $\mathrm{La}_{7} \mathrm{Sr}_{\frac{1}{8}} \mathrm{MnO}_{3}$ compound [40, 66] with those of the $\mathrm{Cr}^{3+}{ }^{8}\left(e_{\mathrm{g}}^{0} t_{2 \mathrm{~g}}^{3}\right)$ ions in $\mathrm{YCrO}_{3}$ compound. The structural parameters of $\mathrm{La}_{\frac{7}{8}} \mathrm{Sr}_{\frac{1}{8}} \mathrm{MnO}_{3}$ compound [40] were from a neutron-powder diffraction study on samples pulverized from a single crystal. This is thus comparable to the results from the present study. Both bond lengths of Mn-O21 and Mn-O22 [Fig. 10(a)] and the bond angle of Mn-O1-Mn [Fig. 12(a)] respond readily to the Jahn-Teller effect that occurs in $\mathrm{La}_{\frac{7}{8}} \mathrm{Sr}_{\frac{1}{8}} \mathrm{MnO}_{3}$ compound within a temperature range of $\stackrel{\frac{8}{8}}{\sim} 180^{\frac{1}{8}}-270 \mathrm{~K}$, whereas, those in $\mathrm{YCrO}_{3}$ compound keep nearly constants, and both Cr$\mathrm{O}$ bond lengths [Fig. 10(b)] and $\mathrm{Cr}-\mathrm{O}-\mathrm{Cr}$ bond angles [Fig. 12(b)] exhibit no response to the AFM transition, consistent with the fact that $\mathrm{Cr}^{3+}$ ions don't have the Jahn-Teller effect. The values of $\mathrm{Cr}-\mathrm{O} 1$ bond lengths are similar to those of the Mn-O21, and $\mathrm{Cr}-\mathrm{O} 21$ and $\mathrm{Cr}-\mathrm{O} 22$ to Mn-O1 (Fig. 10). 
As shown in Figs. 11 and 12 , in contrast to the bond angle of Mn-O-Mn, $\angle \mathrm{Cr}-\mathrm{O}-\mathrm{Cr}$ decreases hugely by $\sim 15^{\circ}$, which in our opinion corresponds intimately to a possible lowering of the crystalline symmetry in the $\mathrm{YCrO}_{3}$ single crystal. The relatively shorter bond length of $\langle\mathrm{Y}-\mathrm{O}\rangle$ (Fig. 13) introduces an immense mismatch between $\mathrm{Y}^{3+}$ and $\mathrm{Cr}^{3+}$ sites, leading to a huge chemical pressure and driving the subsequent rotating and tilting of the $\mathrm{CrO}_{6}$ octahedra.

\section{CONCLUSIONS}

In summary, we have grown a nearly stoichiometric $\mathrm{Y}_{0.97(2)} \mathrm{Cr}_{0.98(2)} \mathrm{O}_{3.00(2)}$ single crystal by a laser diode $\mathrm{FZ}$ furnace. There are three electrons locating on the $3 d t_{2 \mathrm{~g}}$ orbitals of $\mathrm{Cr}^{3+}$ ions, therefore, the $\mathrm{YCrO}_{3}$ compound is a robust insulator. Although the measurements of the applied-magnetic-field dependent heat capacity as well as the magnetization versus temperature and appliedmagnetic field show the character of a very soft ferromagnet with a coersive force of $\sim 0.05 \mathrm{~T}$, the extracted PM CW temperature, $\theta_{\mathrm{CW}}=-433.2(6) \mathrm{K}$, by the fit with a $\mathrm{CW}$ law is strongly negative with the frustrating parameter $f=\left|\theta_{\mathrm{CW}}\right| / T_{\mathrm{N}}=3.061(5)$, and the measured magnetization at $2 \mathrm{~K}$ and $7 \mathrm{~T}$ is only $\sim 3.2 \%$ of the theoretical saturation moment. These indicate that the spin moments of $\mathrm{Cr}^{3+}$ ions in $\mathrm{YCrO}_{3}$ compound are magnetically frustrated. The consistency between the effective PM moment, $\mu_{\text {eff_meas }}=3.95(2) \mu_{\mathrm{B}}$, and the theoretically-calculated value, $\mu_{\text {eff_theo }}=3.873 \mu_{\mathrm{B}}$, validates our results concluded in the framework of the CW-law fitting. By magnetization measurements, we determined the magnetic phase transition temperature as $T_{\mathrm{N}}=141.5(1) \mathrm{K}$ at an applied-magnetic field of $0.01 \mathrm{~T}$. This is in agreement with our neutron-powder and singlecrystal diffraction studies. The magnetic transition temperature was pushed upward to $T_{\mathrm{N}}(5 \mathrm{~T})=144.5(1) \mathrm{K}$ at $5 \mathrm{~T}$, increased by $\sim 3 \mathrm{~K}$.

With our neutron-powder diffraction study, we have established an AFM structure with the propagation vector at $\mathbf{k}=\left(\begin{array}{lll}1 & 1 & 0\end{array}\right)$ and the same unit cell as that of the crystalline structure (with space group Pnma). The direction of the $\mathrm{Cr}^{3+}$ spin moments is along the crystallographic $c$ axis. The refined moment size is $2.45(6)$ $\mu_{\mathrm{B}}$ at $12 \mathrm{~K}, \sim 82 \%$ of the theoretical saturation value $3 \mu_{\mathrm{B}}$. This is consistent with the fact that a magnetic frustration exists in $\mathrm{YCrO}_{3}$ compound. By fitting integrated intensities of the magnetic Bragg (1 110$)$ reflection extracted from the pure magnetic contribution with a power law, we found that the $\mathrm{Cr}^{3+}$ spin interactions were probably two-dimensional Ising like within the reciprocal ( $\left.\begin{array}{lll}1 & 1 & 0\end{array}\right)$ scattering plane. Above $T_{\mathrm{N}}=141.5(1)$ $\mathrm{K}$, the refined lattice constants $a, b$, and $c$, as well as the unit-cell volume $V$, agree well with the Grüneisen rules at zero pressure with a second-order fashion. By com- parison, below $T_{\mathrm{N}}$, the lattice configuration $(a, b, c$, and $V$ ) deviates largely downward from the Grüneisen law, displaying an anisotropic magnetostriction effect along the crystallographic $a, b$, and $c$ axes and a magnetoelastic effect with the unit-cell volume $V$. Especially, upon cooling, the sample contraction is enhanced below $T_{\mathrm{N}}$.

In the whole studied temperature range of $12-302 \mathrm{~K}$, we did not find any crystalline structural phase transition with the neutron-powder diffraction study, whereas by our single-crystal neutron diffraction study, we observed clearly the existence of the Bragg (1 110$)$ peak above the magnetic phase transition temperature 141.5(1) K. This peak persists up to $300 \mathrm{~K}$ and is forbidden by the crystalline orthorhombic structure (with space group Pnma). This implies that the actual crystalline structure of $\mathrm{YCrO}_{3}$ compound is probably lower than the present one. To figure out the reasons for a possible lowering of the structural symmetry in the $\mathrm{YCrO}_{3}$ single crystal, we compared the $t_{2 \mathrm{~g}} \mathrm{YCrO}_{3}$ and the $e_{\mathrm{g}} \mathrm{La}_{7 / 8} \mathrm{Sr}_{1 / 8} \mathrm{MnO}_{3}$ single crystals. It is pointed out that with a limited number of the observed magnetic Bragg peaks, it is hard to determine the canting degree of the AFM Cr ${ }^{3+}$ spins. This can be addressed by a further time-of-flight single-crystal neutron-diffraction study. To determine the actual crystalline structure is not an easy job, but it would shed light on the dielectric anomaly of $\mathrm{YCrO}_{3}$ compound.

\section{ACKNOWLEDGEMENTS}

T.L. acknowledges the National Natural Science Foundation of China (Grant No. 11604214), the Foundation of Department of Education of Guangdong Province (Grant No. 2018KTSCX223), and the Foundation of Department of Science and Technology of Guangdong Province (Grant No. 2020A1515010814). J.M. acknowledges the National Science Foundation of China (Grant No. 11974042). D.O. acknowledges financial support from the Science and Technology Development Fund, Macao SAR (File No. 0029/2018/A1). H.-F.L. acknowledges the University of Macau (Files No. SRG2016-00091-FST and No. MYRG2020-00278-IAPME), the Science and Technology Development Fund, Macao SAR (Files No. 063/2016/A2, No. 064/2016/A2, No. 028/2017/A1, and No. 0051/2019/AFJ), and the Guangdong-Hong KongMacao Joint Laboratory for Neutron Scattering Science and Technology. This research used resources at the Spallation Neutron Source, a Department of Energy Office of Science User Facility operated by Oak Ridge National Laboratory.

* These three authors contributed equally.

† zktang@um.edu.mo 
$\ddagger$ hezb@sustc.edu.cn

$\S$ haifengli@um.edu.mo

[1] O. Auciello, J. F. Scott, and R. Ramesh, Phys. Today 51, 22 (1998).

[2] B. B. Vanaken, T. T. M. Palstra, A. Filippetti, and N. A. Spaldin, Nat. Mater. 3, 164 (2004).

[3] H. J. Zhao, W. Ren, Y. Yang, J. Íñiguez, X. M. Chen, and L. Bellaiche, Nat. Commun. 5, 4021 (2014).

[4] A. S. Gibbs, K. S. Knight, and P. Lightfoot, Phys. Rev. B 83, 094111 (2011).

[5] M. Kenzelmann, A. B. Harris, S. Jonas, C. Broholm, J. Schefer, S. B. Kim, C. L. Zhang, S.-W. Cheong, O. P. Vajk, and J. W. Lynn, Phys. Rev. Lett. 95, 087206 (2005).

[6] M. V. Mostovoy, Phys. Rev. Lett. 96, 067601 (2006).

[7] J. T. Looby and L. Katz, J. Am. Chem. Soc. 76, 6029 (1954).

[8] S. Geller and E. A. Wood, Acta Cryst. 9, 563 (1956).

[9] M. Ardit, G. Cruciani, M. Dondi, M. Merlini, and P. Bouvier, Phys. Rev. B 82, 064109 (2010).

[10] M. V. Kuznetsov and I. P. Parkin, Polyhedron 17, 4443 (1998).

[11] K. Poplawski, K. Schnitzlein, J. Lichtenberger, F. J. Keil, and M. D. Amiridis, Catal. Today 62, 329 (2000).

[12] D. Westphal, A. Laske, S. Jakobs, and U. Guth, Ionics 6, 346 (2000).

[13] H. Li, Synthesis of CMR manganites and ordering phenomena in complex transition metal oxides (Forschungszentrum Jülich GmbH Press, Jülich, 2008).

[14] Y. Zhu, S. Wu, B. Tu, S. Jin, A. Huq, J. Persson, H. S. Gao, D. Ouyang, Z. B. He, D.-X. Yao, Z. K. Tang, and H.-F. Li, Phys. Rev. B 101, 014114 (2020).

[15] K. Sardar, M. R. Lees, R. J. Kashtiban, J. Sloan, and R. I. Walton, Chem. Mater. 23, 48 (2011).

[16] J. Prado-Gonjal, R. Schmidt, J.-J. Romero, D. Ávila, U. Amador, and E. Morán, Inorg. Chem. 52, 313 (2013).

[17] W. J. Weber, C. W. Griffin, and J. L. Bates, J. Mater. Res. 1, 675 (1986).

[18] G. F. Carini II, H. U. Anderson, D. M. Sparlin, and M. M. Nasrallah, Solid State lonics 49, 233 (1991).

[19] M. Kagawa, Y. Kato, and Y. Syono, J. Aeroso Sci. 28, 475 (1997).

[20] J. H. Kim, H.-S. Shin, S.-H. Kim, J.-H. Moon, and B.-T. Lee, Jpn. J. Appl. Phys. 42, 575 (2003).

[21] J. Seo, Y. Ahn, and J. Y. Son, Ceram. Int. 41, 12471 (2015).

[22] V. Bedekar, R. Shukla, and A. K. Tyagi, Nanotechnology 18, 155706 (2007).

[23] J. Bahadur, D. Sen, S. Mazumder, R. Shukla, and A. K. Tyagi, J. Phys. Condens. Mat. 20, 345201 (2008).

[24] R. Sinha, S. Basu, and A. K. Meikap, Phys. E LowDimens. Syst. Nanostruct. 113, 194 (2019).

[25] V. M. Jüdin and A. B. Sherman, Solid State Commun. 4, 661 (1966).

[26] E. F. Bertaut, G. Bassi, G. Buisson, P. Burlet, J. Chappert, A. Delapalme, J. Mareschal, G. Roult, R. Aleonard, R. Pauthenet, and J. P. Rebouillat, J. Appl. Phys. 37, 1038 (1966).

[27] T. Morishita and K. Tsushima, Phys. Rev. B 24, 341 (1981).

[28] A. Durán, A. M. Arévalo-López, E. Castillo-Martínez, M. García-Guaderrama, E. Moran, M. P. Cruz, F. Fernández, and M. A. Alario-Francob, J. Solid State
Chem. 183, 1863 (2010).

[29] L. Hao, L. Yang, M.-H. Lee, T.-H. Lin, Z. Zhang, X. Xie, and H. Zhu, J. Alloys Compd. 601, 14 (2014).

[30] S. Sidi Ahmed, G. D. Ngantso, M. Boujnah, A. Benyoussef, and A. El Kenz, Phys. Lett. A 383, 121 (2019).

[31] C. R. Serrao, A. K. Kundu, S. B. Krupanidhi, U. V. Waghmare, and C. N. R. Rao, Physical Review B 72, 220101(R) (2005).

[32] L. Katz, Acta Cryst. 8, 121 (1955).

[33] J. P. Van Der Ziel and L. G. Van Uitert, Phys. Rev. 179, 343 (1969).

[34] J. P. Remeika, J. Am. Chem. Soc. 78, 4259 (1956).

[35] W. H. Grodkiewicz and D. J. Nitti, J. Am. Ceram. Soc. 49, 576 (1966).

[36] N. D. Todorov, M. V. Abrashev, V. G. Ivanov, G. G. Tsutsumanova, V. Marinova, Y.-Q. Wang, and M. N. Iliev, Phys. Rev. B 83, 224303 (2011).

[37] S. Sugano, K. Aoyagi, and K. Tsushima, J. Phys. Soc. Jpn. 31, 706 (1971).

[38] Y. H. Zhu, S. Wu, Z. K. Tang, and H.-F. Li, A method of centimeter-sized single crystal growth of chromate compounds and related storage device, China Patent No. 201911281088.8, (2019).

[39] S. Wu, Y. H. Zhu, H. S. Gao, Y. Xiao, J. C. Xia, P. F. Zhou, D. F. Ouyang, Z. Li, Z. Q. Chen, Z. K. Tang, and H.-F. Li, ACS Omega 5, 16584 (2020).

[40] H.-F. Li, Y. Su, Y. G. Xiao, J. Persson, P. Meuffels, and Th. Brückel, Eur. Phys. J. B 67, 149 (2009).

[41] Y. Xiao, Y. Su, H.-F. Li, C. M. N. Kumar, R. Mittal, J. Persson, A. Senyshyn, K. Gross, and Th. Brueckel, Phys. Rev. B 82, 094437 (2010).

[42] Q. Zhang, W. Tian, H. Li, J.-W. Kim, J. Yan, R. W. McCallum, T. A. Lograsso, J. L. Zarestky, S. L. Bud'ko, R. J. McQueeney, and D. Vaknin, Phys. Rev. B 88, 174517 (2013)

[43] W. H. Ji, L. Yin, W. M. Zhu, C. M. N. Kumar, C. Li, H.F. Li, W. T. Jin, S. Nandi, X. Sun, Y. Su, Th. Brückel, Y. Lee, B. N. Harmon, L. Ke, Z. W. Ouyang, and Y. Xiao, Phys. Rev. B 100, 134420 (2019).

[44] J. Rodríguez-Carvajal, Phys. B 192, 55 (1993).

[45] N. N. Greenwood and A. Earnshaw, ChemistryoftheElements (Elsevier Ltd, Oxford, 1997).

[46] A. P. Ramirez, Handbookof MagneticMaterials, Vol. 13, edited by K. Buschow (Elsevier, Amsterdam, 2001), p. 423.

[47] A. P. Ramirez, Annu. Rev. Mater. Sci. 24, 453 (1994).

[48] H. T. Diep, FrustratedSpinSystems (World Scientific, Singapore, 2004).

[49] M. P. Shores, E. A. Nytko, B. M. Bartlett, and D. G. Nocera, J. Am. Chem. Soc. 127, 13462 (2005).

[50] H.-F. Li, C. Zhang, A. Senyshyn, A. Wildes, K. Schmalzl, W. Schmidt, M. Boehm, E. Ressouche, B. Hou, P. Meuffels, G. Roth, and Th. Brückel, Front. Phys. 2, 42 (2014).

[51] H.-F. Li, A. Senyshyn, O. Fabelo, J. Persson, B. Hou, M. Boehm, K. Schmalzl, W. Schmidt, J.-P. Vassalli, P. Thakuria, X. Sun, L. Wang, G. Khazaradze, B. Schmitz, C. Zhang, G. Roth, J. G. Roca, and A. Wildes, J. Mater. Chem. C 3, 7658 (2015).

[52] H.-F. Li, npj Comput. Mater. 2, 16032 (2016).

[53] A. H. Cooke, D. M. Martin, and M. R. Wells, J. Phys. C: Solid State Phys. 7, 3133 (1974).

[54] W. L. Stephen, Theoryofneurtronscattering fromcondensedmatter (Clarendon Press, Oxford, 1984). 
[55] C. Kittel, QuantumTheoryof Solids (Wiley, New York, 1987).

[56] H. F. Li, Y. Su, J. Persson, P. Meuffels, J. M. Walter, R. Skowronek, and Th. Brückel, J. Phys.: Condens. Matter 19, 016003 (2006).

[57] H. F. Li, Y. Su, J. Persson, P. Meuffels, J. M. Walter, R. Skowronek, and Th. Brückel, J. Phys.: Condens. Matter 19, 176226 (2007).

[58] A. Tari, TheSpecificHeatof MatteratLowTemperatures (Imperial College Press, London, 2003).

[59] H.-F. Li, A. Wildes, B. Y. Hou, C. Zhang, B. Schmitz, P. Meuffels, G. Rothb, and Th. Brückel, RSC Adv. 4, 53602 (2014)
[60] M. F. Collins, MagneticCriticalScattering (Oxford University Press, London, 1989).

[61] S. J. Payne, M. E. Hagen, and M. J. Harris, J. Phys.: Condens. Matter 891 (1996).

[62] H.-F. Li, Y. Xiao, B. Schmitz, J. Persson, W. Schmidt, P. Meuffels, G. Roth, and Th. Brückel, Sci. Rep. 2, 750 (2012).

[63] D. C. Wallace, Phys. Rev. B 58, 15433 (1998).

[64] L. Vočadlo, K. S. Knight, G. D. Price, and I. G. Wood, Phys. Chem. Miner. 29, 132 (2002).

[65] H.-F. Li, J. Q. Yan, J. W. Kim, R. W. McCallum, T. A. Lograsso, and D. Vaknin, Phys. Rev. B 84, 220501(R) (2011).

[66] A. Kotani, H. Nakajima, K. Harada, Y. Ishii, and S. Mori, Phys. Rev. B 95, 144403 (2017). 